\title{
Preventive and Restorative Responses of Broccoli Sprouts on Biomechanical Structures, Histomorphometric, and Gene Expression Levels of Degenerated Intervertebral Disc in Rabbit Model
}

\author{
Babatunde Ogunlade ${ }^{1}$, Obinna P. Fidelis ${ }^{2}$, Sunday Aderemi Adelakun ${ }^{1}$ \\ ${ }^{1}$ Human Anatomy Department, Federal University of Technology Akure, Akure, Nigeria \\ ${ }^{2}$ Biomedical Technology Department, Federal University of Technology Akure, Akure, Nigeria
}

\begin{abstract}
Study Design: Symptomatic disk degeneration is characterized by early pathological changes in the morphological, biochemical, and biomechanical properties of the intervertebral disc (IVD). Several methods that can be used to resolve this condition are being researched.

Purpose: The present study was designed to examine the preventive and restorative properties of broccoli sprouts extracts in an annular puncture rabbit model of IVD degeneration.

Overview of Literature: IVD degeneration is closely associated with low back pain. A degenerated IVD is cytoarchitecturally characterized by a reduction in cell number, morphological changes in the disk tissue, and changes in the extracellular matrix contents. Methods, such as imaging and biochemical characterization, have been used for distinguishing between degenerated disk and nondegenerated disk.

Methods: Twenty New Zealand white rabbits (weighing approximately 2.0-3.5 kg) were divided into four groups ( $n=5)$. Group 1 received $50 \mathrm{mg} / \mathrm{kg}$ of normal saline solution orally for 4 weeks; group 2 received $50 \mathrm{mg} / \mathrm{kg}$ of normal saline solution immediately after puncture for 4 weeks; group 3 received $300 \mathrm{mg} / \mathrm{kg}$ of aqueous extract of broccoli sprouts orally immediately after puncture for 4 weeks; and group 4 received $300 \mathrm{mg} / \mathrm{kg}$ of aqueous extract of broccoli sprouts after 4 weeks of puncture for another 4 weeks. After the experiment, the disk height index (DHI), histomorphometry, and gene expression level were measured and analyzed.

Results: The photomicrograph showed that the histological features of the punctured-treated groups (groups 3 and 4) were more similar to those of the non-punctured group than those of the punctured non-treated group. There was a significant difference in the $\mathrm{DHI}$ and relative gene expression levels among the non-treated punctured groups compared with those of the non-punctured and punctured-treated groups, respectively.

Conclusions: This study shows that the aqueous extract of broccoli sprouts exerts preventive and restorative effects in a rabbit model with disk degeneration.
\end{abstract}

Keywords: Broccoli sprouts; Disk degeneration; Disk height; Disk puncture; Gene expression; Histomorphology

Received Sep 17, 2020; Revised Oct 26, 2020; Accepted Nov 1, 2020

Correspondence author: Obinna P. Fidelis

Biomedical Technology Department, Federal University of Technology Akure, Akure, Ondo State, Nigeria

Tel: +234-80-381-937-99, Fax: +234-80-363-187-57, E-mail: opfidelis@futa.edu.ng 


\section{Introduction}

Intervertebral discs (IVDs) are partially movable secondary cartilaginous joints that connect each of the vertebral bodies in the spine and function for load transfer as well as mobility [1]. Disk degeneration occurs as an early pathological change in the morphological, biochemical, and biomechanical properties of the IVD [2]. Previous experimental studies on disk degeneration with animal models have provided a deeper understanding of the pathological mechanism of disk degeneration as well as the testing of therapeutic mediations [3]. Although several experimental injury models that used animals exist [4], the most commonly used method is that of multiple needle puncture to the IVD [5]. A degenerated IVD is cytoarchitecturally characterized by a reduction in the cell number, morphological changes in the disk tissue, and changes in the extracellular matrix contents [6]. Several studies have used methods, such as imaging and biochemical characterization, for making a distinction between degenerated disk and non-degenerated disk [7].

IVD degeneration is strongly associated with low back pain [8] caused by several factors that may include the use of non-ergonomic and poorly designed furniture and/ or workplace factors [9]. IVD degeneration begins with changes to the cellular microenvironment within the substructures of the disk and progresses over time, resulting in structural breakdown and functional impairment of the disk tissue [8]. Several factors, such as altered mechanical loading [10], reduced nutrient supply, and hereditary factors [11], are closely related to the progression of the disk degenerative cascade. Excessive loading (usually in a form of compressive force) can result in localized tissue injury that takes a long time to repair and alters the strain distribution throughout the extracellular matrix of the entire disk [10].

The scarcity of the degenerated human disk tissue and the almost nonexistence of healthy tissue for comparison in in-vitro studies necessitates the use of animal models for investigating IVD degeneration [1]. Animal models are favorably used for these studies because the disks of both large and small animals possess anatomical and biomechanical traits that make them suitable models for studying the human tissue [12]. Techniques for initiating degenerative changes in such model systems include annulus fibrosus (AF) injury [13], mechanical overload [14], and enzymatic treatment that would enable a reduction in the nucleus pulposus (NP) glycosaminoglycan content [15].

Broccoli sprout is an annual fast-growing plant belonging to the family brassica oleracea native to the Asia and Mediterranean and contains alkaloids, flavonoids, tannins, polyphenols, essential oils, phenolics, and polypeptides as well as other antioxidant-promoting properties; furthermore, it has a negligible amount of indole glucosinolates (indole-3-carbinol) that can enhance tumorigenesis, thereby protecting the body against cancer [16]. Studies show the phytotherapeutic potential of broccoli sprouts in skin diseases, cancer, diabetes, etc. The most potent phytonutrient of broccoli sprout (sulforaphane) suppresses nasal inflammatory response; prevents neurodegeneration, neuronal loss, and oxidative stress; and protects chondrocyte-like cells from apoptosis [16,17]. Given the health implications of IVD degeneration, this study was designed to examine the preventive and restorative properties of aqueous extract of broccoli sprouts on NP cell apoptosis in an annular puncture rabbit model of IVD degeneration.

\section{Materials and Methods}

\section{Collection of plant material}

Fresh broccoli sprouts were obtained from Akure town, Ondo state, Nigeria. The plant sample was authenticated at the Department of Pure and Applied Biology, Ladoke Akintola University of Technology, Ogbomoso, Nigeria.

\section{Preparation of the plant extract}

The samples were thoroughly washed in sterile water and milled using an automatic electric blender (model FS-323, China) to extract the juice using cheesecloth and Whatman \#1 filter paper; $500 \mathrm{~g}$ of the filtrate was concentrated using a rotary evaporator (Rotavapor R-210; Büchi, Flawil, Switzerland) at $42^{\circ} \mathrm{C}-47^{\circ} \mathrm{C}$.

\section{Surgical technique}

All the animal handling and surgical procedures were conducted after obtaining ethical approval from the Health Research Ethics Committee, College of Medicine of the University of Lagos (approval no., CM/ HREC/07/2019/121). Twenty New Zealand white rabbits 
(weighing approximately $2.0-3.5 \mathrm{~kg}$ ) were used with the approval of the institutional animal care committee's approval. The rabbits were sacrificed 4 weeks after the puncture and a total of 8 weeks after the beginning of the experiment. The surgical technique was performed using the modified procedure of Ogunlade et al. [17]. Each rabbit was anesthetized with xylazine $(5 \mathrm{mg} / \mathrm{kg})$ and ketamine $(35 \mathrm{mg} / \mathrm{kg}$ ), and the fur was shaved from the mid-back and right flank. A lateral plain radiograph was obtained to establish the pre-injection baseline height of the IVDs. The L5-L6 disk was identified via manual palpation of the interspinous space from the mid-back and the pelvic rim; thereafter, a $21 \mathrm{G}$ angiography needle was inserted 3-4 $\mathrm{cm}$ ventrally from the midline into the disk space and held in the disk space for 30 seconds. In each rabbit, each of the three disks (L3-L4, L4-L5, and L5-L6) was punctured. The L1-L2 and L2-L3 levels were designated as the non-punctured, internal controls. For each level, all the procedures for identification and puncture were performed within 5 minutes. Special care was taken to minimize contact with the periosteal tissues of the vertebrae because this could cause hypertrophy of the soft tissues and bony structures around the disks; however, this was not observed. The rabbits were monitored for neurological symptoms. The rabbits were placed in their cages after observation for recovery.

The rabbits were divided into four groups, with five animals per group: (1) group 1 received $50 \mathrm{mg} \mathrm{NaCl}(5.5$ $\mathrm{mL}$ normal saline solution) per kilogram of rabbit orally for 4 weeks (non-punctured group); (2) group 2 received $50 \mathrm{mg} / \mathrm{kg}$ normal saline solution immediately after the puncture for 4 weeks; (3) group 3 received oral administration of $5.5 \mathrm{~mL}$ aqueous extract of broccoli sprouts per kilogram of rabbit immediately after the puncture for 4 weeks; and (4) group 4 received oral administration of 5.5 $\mathrm{mL}$ aqueous extract of broccoli sprouts per kilogram of rabbit after 4 weeks of puncture for another 4 weeks.

Lateral radiograph of the lumbar spine was taken before the disk puncture and just before the animals were sacrificed for measuring the IVD height. The rabbits were allowed free access to water and fed a restricted diet of standard pelletized rat chow. After the experiments, the rabbits were euthanatized, and the IVDs were evaluated.

\section{Determination of the percentage disk height index}

Lateral plain radiographs of the lumbar spine were scanned and digitally stored using a radiograph machine by a veterinary radiologist (collimator-to-film distance, 50 $\mathrm{cm}$; exposure, $5 \mathrm{mAs}$; penetration power, $44 \mathrm{kVp}$ ). During the radiography, special care was taken to minimize the axial rotation of the disk space by holding the rabbit in the lateral decubitus position while ensuring that the radiograph beam was maintained perpendicular to the sagittal plane. Moreover, each rabbit was treated with a consistent amount of ketamine $(35 \mathrm{mg} / \mathrm{kg})$ and intramuscular injection of xylazine $(5 \mathrm{mg} / \mathrm{kg})$ to provide a similar degree of muscle relaxation for minimizing the differences in the disk height. Vertebral body height and disk height were measured using Scion Imaging Software 4.0 (Scion Corp., Chicago, IL, USA) and analyzed using an image analysis program (Image J; National Institutes of Health, Bethesda, MD, USA).

The IVD height was expressed as the disk height index (DHI) using the method described by Ogunlade et al. [17]. The DHI was calculated by averaging the measurements obtained from the anterior, middle, and posterior portions of the IVD and dividing by the average of the adjacent vertebral body heights. Changes in the DHI were expressed as percentage DHI and normalized to the measured preoperative IVD height (percentage DHI=postoperation DHI/preoperation DHI $\times 100)$.

\section{Histological studies}

Histomorphology and collagen fibers were analyzed using the procedure described by Ogunlade et al. [17]. The rabbits were sacrificed after the experiment was completed at different periods for the histological evaluation. The intact specimens, including the AF, the NP, both endplates (EP), and the adjacent vertebral body bone, were fixed. The tissues were fixed with $10 \%$ neutral buffered formalin for 48 hours, decalcified in decalcification solution (ethylenediaminetetraacetic acid; National Diagnostics, Atlanta, GA, USA) for 3 days, and then processed for paraffin sectioning. The sections were stained with hematoxylin and eosin to determine the morphology of the AF, NP, and EP and the number of chondrocyte-like cells within the NP, inner AF (IAF), and outer AF (OAF); hematoxylin-van Gieson stain was used to demonstrate the collagen fibers in AF. The slides were analyzed with light microscope Image J software (National Institutes of Health). The degree of IVD degeneration was evaluated by blinded observers (2) using Boo's histological grading scale [18]. 


\section{Biochemical analysis}

Two rabbits were randomly chosen for real-time quantitative polymerase chain reaction (PCR) as per the procedure described by Ogunlade et al. [17]. The L3L4 and L4-L5 levels were extracted for the punctured disk, whereas the L1-L2 disk was extracted as a nonpunctured control. From each disk, the NP was carefully removed from AF and stored separately. The tissues were immediately placed in liquid nitrogen and frozen at $-80^{\circ} \mathrm{C}$ in preparation for the PCR analysis. The frozen NP samples were homogenized using Mini-Beadbeater (Bio Spec, Bartlesville, OK, USA) in $1 \mathrm{~mL}$ Trizol reagent (Invitrogen, Carlsbad, CA, USA). RNA was extracted as per the manufacturer's instructions. The primers for the rabbit-specific genes were designed as per the published sequences in the procedure of Young-Joon (2013). Gene expression was analyzed using reverse transcription-PCR with ABI PRISM 9700 (Applied Biosystems, Foster City, CA, USA). Gene expression levels were calibrated using a constitutively expressed housekeeping gene, glyceraldehyde phosphate dehydrogenase. A positive standard curve for each primer was obtained with a serially diluted cDNA sample mixture. Quantifications of gene expression were performed (comparative Ct method) with standard curves and normalized to GAPDH (glyceraldehyde 3-phosphate dehydrogenase) in each sample; thereafter, the expression of the treated disks was normalized to the level observed in the control disks.

The sequences were as follows: aggrecan (5' GCTACGGAGACAAG GATGAGTTC $3^{\prime}$ and 5' CGTAAAAGACCTCACCCTCCAT $3^{\prime}$ ), matrix metalloproteinase-13 (5' TGCCCCTCCTCAACAGTAAC $3^{\prime}$ and 5' GAGCCCGCTGCATTCTTCTT 3'), type II collagen ( $5^{\prime}$ TCAGGAATTTGGTGTGGACATA $3^{\prime}$ and 5' CCGGACTGTGAGGTTAGGATAG 3'), type I collagen (5' GGGCAAGACAGTCATCGAATA $3^{\prime}$ and 5' GATTGGGATGGAGGGAGTTTA 3'), GAPDH (5' AAGGCCATCACCATCTTCCA $3^{\prime}$ and 5' GGATGCGTTGCTGACAATCT 3').

\section{Statistical analyses}

Data were analyzed using the IBM SPSS ver. 24.0 (IBM Corp., Armonk, NY, USA). Longitudinal radiography data were analyzed using two-way analysis of variance followed by multiple comparisons with the Bonferroni method.
Quantitative real-time PCR data were analyzed using the Student $t$-test. Data are presented as mean \pm standard error of mean values. The level of significance was set at $p<0.05$.

\section{Results}

\section{Radiological analysis}

The percentage disk height of the punctured group administered with normal saline showed a significant decrease as compared with that of the non-punctured group (about $31 \%$ decrease as compared to that at baseline, $p<0.05$ ), as shown in Fig. 1. The percentage DHI of the treated group (immediately and 4 weeks after the puncture) was significantly different from that of group $2(p<0.05)$, as shown in Fig. 1 . The percentage disk height of the group that was administered broccoli sprouts extracts 4 weeks after the puncture was significantly decreased as compared with that of the group that was administered broccoli sprouts immediately after the puncture (group 3$)(p<0.05)$ as in Fig. 1. There were no significant differences in the percentage DHI among the treated animals that received broccoli sprouts extract immediately after the puncture compared with the non-punctured group (group 1).

\section{Histological analyses}

The cytoarchitectural section for the treated group that was administered the aqueous extract of broccoli sprouts showed better-maintained morphology of the IVD than the punctured group (group 2). The photomicrograph

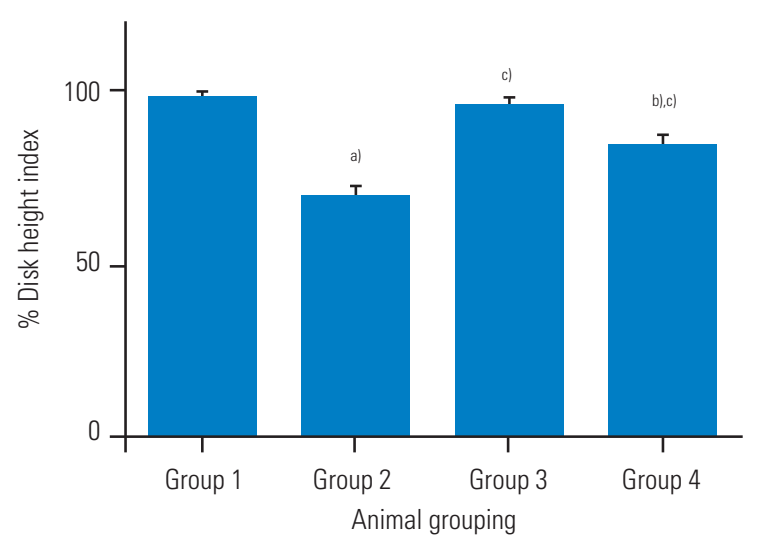

Fig. 1. Intervertebral disc height after administration ofaqueous extract of broccoli sprouts immediately and 4 weeks after puncture. Values are presented as mean \pm standard error of mean for five rabbits in each group. ${ }^{\text {al }} p<0.05$ as compared to group 1. ${ }^{\text {b) }} p<0.05$ as compared to group $3 .{ }^{c)} p<0.05$ as compared to group 2. 
of the non-punctured group (positive control) showed normal disk morphology with numerous chondrocytelike cells confined within the NP (Fig. 2). However, the punctured non-treated group revealed structural distortion in the cytoarchitecture of the disk that resembled degenerated disks, such as collapses of the AF, extrusion of the chondrocytes-like cells through the fissures, and decrease in the disk height. The photomicrograph of the punctured-treated groups (groups 3 and 4) administered the aqueous extract of broccoli sprouts showed histological features similar to those in the non-punctured group.

\section{Histological grading scores}

The observatory grading scores from the photomicrograph results were assigned as per a previously described histological grading score for IVD degeneration [19]. The scoring grade ranges from 4 (normal) to 12 (severely degenerated), depending on the degeneration level. In the present study, there were no differences in the scores of the treated groups (groups 3 and 4) that received aqueous extract of broccoli sprouts and the non-punctured control (group 1) (Fig. 3). The degeneration was lesser in the treated groups (groups 3 and 4 ) than in group 2 (Fig. 3).
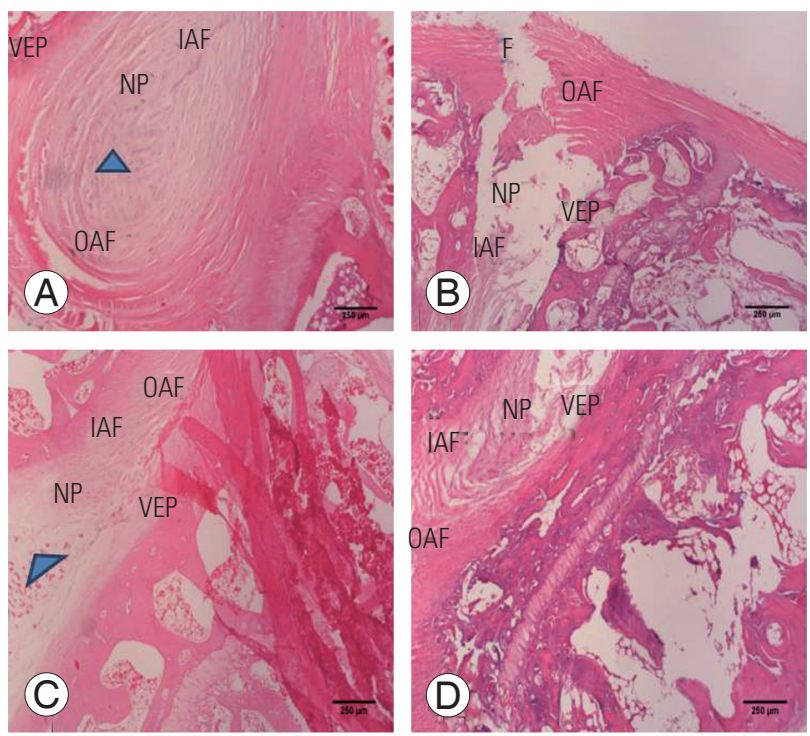

Fig. 2. Photomicrograph of intervertebral disc (IVD) histology in rabbit administered with aqueous extract of broccoli sprouts immediately after puncture and 4 weeks after puncture $(H \& E, \times 100)$. (A) Group 1: showing normal IVD cytoarchitecture with numerous chondrocyte-like cells (arrow). (B) Group 2: showing presence of fissure (F) and extrusion of the nucleus pulposus (NP) contents via the fissures. (C) Group 3: showing nearly similar features with group 1. (D) Group 4: showing restoration of NP contents. VEP, vertebral end plate; IAF, inner annulus fibrosus; $\mathrm{OAF}$, outer annulus fibrosus.

\section{Morphometry analysis of chondrocyte-like cells in intervertebral disc}

In this study, the number of chondrocyte-like cells in the IAF or in the NP of the animals that were administered aqueous extract of broccoli sprouts is an important cytoarchitectural change. In the NP and IAF, there was an increase in the number of chondrocyte-like cells in the treated groups that received aqueous extract of broccoli sprouts (immediately after the puncture and 4 weeks after the puncture, respectively) compared with that in group $2(p<0.05)$ (Fig. 4). In the OAF, no significant differences were observed among the groups (Fig. 4).

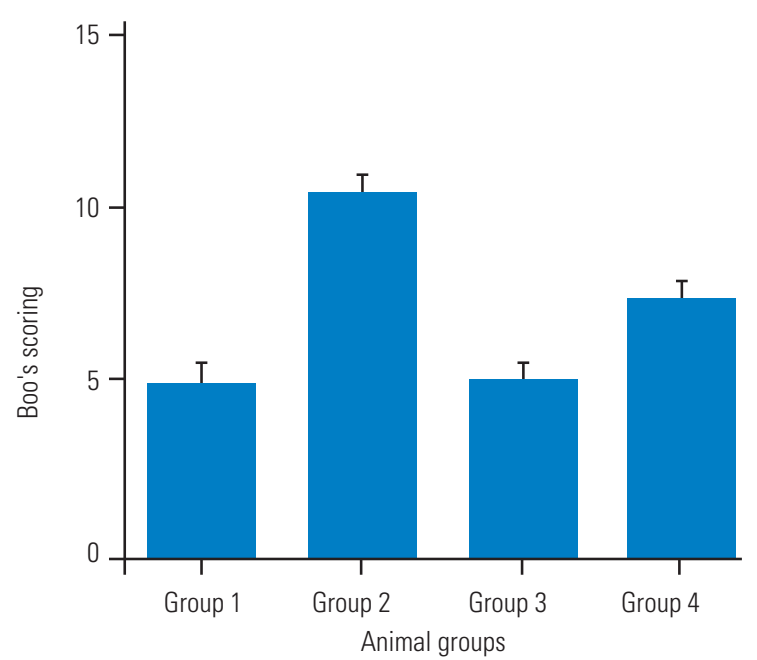

Fig. 3. Histological grading scores for level of degeneration of rabbit intervertebral disc after administration ofaqueous extract of broccoli sprouts immediately and 4 weeks after puncture.

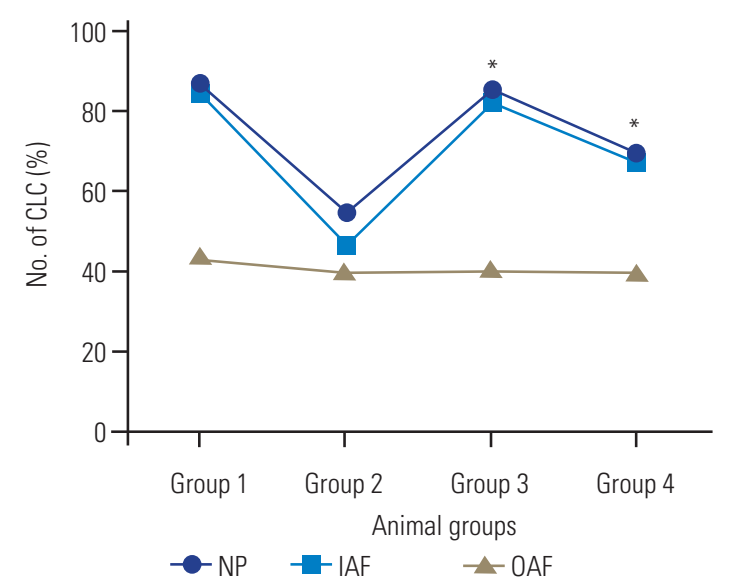

Fig. 4. Number of chondrocyte-like cells (CLC, \%) in nucleus pulposus (NP), inner annulus fibrosus (IAF) and outer annulus fibrosus (OAF) of rabbit intervertebral disc after aqueous extract of broccoli sprouts administration. ${ }^{*} p<0.05$ as compared to group 2. 


\section{Discussion}

In this study, we observed preventive and regenerative response of the oral administration of aqueous extract of broccoli sprouts in annular needle puncture rabbit model of disk degeneration. The results show that the administration of the aqueous extract of broccoli sprouts can prevent and restore the IVD height, disk morphology, and extracellular matrix components of the disk in a rabbit model. The study showed slow progressive degeneration of the IVD overtime as evident by radiological (percentage DHI) and histological (histological grading scores) findings. Therefore, the annular punctured model of disk degeneration is similar to human disk degeneration that shows a progressive decrease in the disk height as the disk ages $[8,17]$. To our knowledge, the use of broccoli for disk regeneration is novel. However, a previous experimental study showed similar morphological features after comparing with the results from this present study [17]. Furthermore, the histomorphological grading system described by Boos et al. [19] in 2002 showed findings similar to those in the present trial and subsequent recovery after broccoli administration.

The present study showed that the administration of aqueous extract of broccoli sprouts immediately after the puncture prevented the progression of disk degeneration as observed in the percentage DHI (Fig. 1). The histological findings in this study showed various disorganizations in the disk morphology, such as discrete disorientation of the NP cells, extrusion of the NP cells, and decrease in lamella disorganization with complete obliteration of its cavity. The cytoarchitecture of the disk tissue showed restoration and prevention of annular disk degeneration in response to the administration of aqueous extract of broccoli sprouts in terms of the degenerated IVD. The morphological findings showed distortion in the disk architecture, expulsion of the disk cells through the fissures in the AF, bulging of the NP into the AF, wavy fibrocartilage lamella, and associated fibrochondrocyte-like cells of the AF that was restored following the administration of the aqueous extract of broccoli sprouts. The avascular NP and AF received nutrients via a diffusion mechanism, such that the active component in broccoli was able to repair and subsequently regenerate the chondrocyte-like cells, thereby preserving the histoarchitecture of the disk. The preventive and regenerative potential of broccoli sprouts observed in the radiology and histomorphology could be attributed to some phytonutrients, such as sulforaphane and glucoraphanin, which, in previous observation, revealed that they inhibit, halt, or reverse neoplastic lesions and increase the survival rates in cancer patients [18].

\section{Conclusions}

The present results showed that the administration of the aqueous extract of broccoli sprouts immediately after the puncture could prevent the progression of disk degeneration and restore the IVD height, disk morphology, and extracellular matrix components of the disk in the rabbit model. The cytoarchitecture of the disk tissue showed the restoration and prevention of annular disk degeneration in response to the administration of the aqueous extract of broccoli sprouts on the degenerated IVD. The morphological findings show distortion in disc architecture, expulsion of the disc cells through the fissures in the AF, bulging of the NP into the AF, wavy fibrocartilage lamella, and associated fibrochondrocyte-like cells of the AF that was restored with the administration of the aqueous extract of broccoli sprouts. These findings provide valuable information for the consideration of clinical trials.

\section{Conflict of Interest}

No potential conflict of interest relevant to this article was reported.

\section{ORCID}

Babatunde Ogunlade: https://orcid.org/0000-0003-29058874; Obinna P. Fidelis: https://orcid.org/0000-0002-65686774; Sunday Aderemi Adelakun: https://orcid.org/00000002-8328-3119

\section{Author Contributions}

Babatunde Ogunlade: study design and conceptualization, data acquisition and analysis, drafting of manuscript and supervision; Obinna P. Fidelis: study conceptualization, drafting and critical revision of manuscript, administrative support; Sunday Aderemi Adelakun: data acquisition and analysis, drafting of manuscript, study design; and all authors carefully read the final manuscript. 


\section{References}

1. Smith LJ, Nerurkar NL, Choi KS, Harfe BD, Elliott DM. Degeneration and regeneration of the intervertebral disc: lessons from development. Dis Mode Mech 2011;4:31-41.

2. Nerlich AG, Schleicher ED, Boos N. 1997 Volvo Award winner in basic science studies: immunohistologic markers for age-related changes of human lumbar intervertebral discs. Spine (Phila Pa 1976) 1997;22:2781-95.

3. Lotz JC. Animal models of intervertebral disc degeneration: lessons learned. Spine (Phila Pa 1976) 2004;29:2742-50.

4. Gruber HE, Johnson T, Norton HJ, Hanley EN Jr. The sand rat model for disc degeneration: radiologic characterization of age-related changes: crosssectional and prospective analyses. Spine (Phila $\mathrm{Pa}$ 1976) 2002;27:230-4.

5. Rousseau MA, Ulrich JA, Bass EC, Rodriguez AG, Liu JJ, Lotz JC. Stab incision for inducing intervertebral disc degeneration in the rat. Spine (Phila Pa 1976) 2007;32:17-24.

6. Vialle EN, Vialle LR, Arruda Ade O. Histomorphometric analysis of experimental disc degeneration. Global Spine J 2012;2:129-36.

7. Omlor GW, Nerlich AG, Wilke HJ, et al. A new porcine in vivo animal model of disc degeneration: response of anulus fibrosus cells, chondrocyte-like nucleus pulposus cells, and notochordal nucleus pulposus cells to partial nucleotomy. Spine (Phila Pa 1976) 2009;34:2730-9.

8. Freemont AJ. The cellular pathobiology of the degenerate intervertebral disc and discogenic back pain. Rheumatology (Oxford) 2009;48:5-10.

9. Cranz G. The Alexander Technique in the world of design: posture and the common chair: part I: the chair as health hazard. J Bodyw Mov Ther 2000;4:90-8.

10. Stokes IA, Iatridis JC. Mechanical conditions that accelerate intervertebral disc degeneration: overload versus immobilization. Spine (Phila Pa 1976) 2004;29:2724-32.
11. Battie MC, Videman T. Lumbar disc degeneration: epidemiology and genetics. J Bone Joint Surg Am 2006;88 Suppl 2:3-9.

12. Beckstein JC, Sen S, Schaer TP, Vresilovic EJ, Elliott DM. Comparison of animal discs used in disc research to human lumbar disc: axial compression mechanics and glycosaminoglycan content. Spine (Phila Pa 1976) 2008;33:E166-73.

13. Elliott DM, Yerramalli CS, Beckstein JC, Boxberger JI, Johannessen W, Vresilovic EJ. The effect of relative needle diameter in puncture and sham injection animal models of degeneration. Spine (Phila Pa 1976) 2008;33:588-96.

14. Kroeber MW, Unglaub F, Wang $\mathrm{H}$, et al. New in vivo animal model to create intervertebral disc degeneration and to investigate the effects of therapeutic strategies to stimulate disc regeneration. Spine (Phila $\mathrm{Pa}$ 1976) 2002;27:2684-90.

15. Boxberger JI, Auerbach JD, Sen S, Elliott DM. An in vivo model of reduced nucleus pulposus glycosaminoglycan content in the rat lumbar intervertebral disc. Spine (Phila Pa 1976) 2008;33:146-54.

16. Ravikumar C. Therapeutic potential of Brassica oleracea (broccoli): a review. Int J Drug Dev Res 2015;7:9-10.

17. Ogunlade B, Osinubi AA, Duru FI, Adelakun SA, Ogunlade SA, Alao AA. Histomorphological response of sulforaphane and riboceine on annular puncture-induced model of rabbits intervertebral disc degeneration. Eur J Biomed Pharm Sci 2017;4:19.

18. Tang L, Zirpoli GR, Guru K, et al. Intake of cruciferous vegetables modifies bladder cancer survival. Cancer Epidemiol Biomarkers Prev 2010;19:1806-11.

19. Boos N, Weissbach S, Rohrbach H, Weiler C, Spratt KF, Nerlich AG. Classification of age-related changes in lumbar intervertebral discs: 2002 Volvo Award in basic science. Spine (Phila Pa 1976) 2002;27:2631-44. 